Submitted to Electrochimica Acta (ISE 2006 Special Issue): 17 November, 2006

\title{
The Effect of Anion Identity on the Viscoelastic Properties of Polyaniline Films During Electrochemical Film Deposition and Redox Cycling
}

\author{
Mohamoud A. Mohamoud and A. Robert Hillman* \\ Department of Chemistry, University of Leicester \\ University Road, Leicester LE1 7RH, UK
}

\begin{abstract}
Acoustic admittance measurements at thickness shear mode resonators were used to determine shear moduli for polyaniline films during their potentiodynamic electrodeposition and subsequent redox cycling in aqueous background electrolyte. Data were acquired for films doped with perchlorate, sulphate or chloride anion. For all media, film shear moduli increased progressively with film thickness, from values consistent with a diffuse fluid-like layer to values typical of a viscoelastic material. At any given thickness, both the storage and loss moduli were largest in perchlorate medium; values in chloride and sulphate media were similar to each other, but smaller than in perchlorate. These measures of polymer dynamics are consistent with a previous classification of polyaniline film behaviour, in which perchlorate-doped films are viewed as compact while chlorideand sulphate-doped films are viewed as more open. In monomer-free background electrolyte solution, both film shear modulus components for all anions increased modestly upon film oxidation. Despite some hysteresis on the timescale of slow scan voltammetry, these variations were chemically reversible. Based on measurements involving deposition from chloride medium and transfer to sulphate medium, film shear moduli respond promptly to changes in dopant identity; this is consistent with rapid redox-driven exchange of anions with the bathing electrolyte.
\end{abstract}

Keywords: Conducting polymer; polyaniline; acoustic wave device; EQCM; viscoelasticity; thin film deposition

* Corresponding author (email address: arh7@le.ac.uk) 


\section{INTRODUCTION}

Polyaniline is a conducting polymer that has attracted considerable research interest [16], notably with regard to its chemical [7], electrochemical [8, 9] and optical [4, 9] properties. In pursuit of understanding these properties based on the underlying elementary processes, detailed studies have been reported of various features of redox switching dynamics [3, 8, 10-14] and polymer structure [3, 10, 15-17]. In contrast, polymer dynamics have received rather less attention; here we focus on this aspect of polyaniline behaviour, via an exploration of film viscoelastic properties.

Quite generally, one might expect electroactive film composition, structure and dynamics to be related. Consequently, the absence of information on any one of these aspects prevents fundamental understanding of film behaviour. As indicated above, the current paucity of information on polymer dynamics is the weak link in this chain of understanding. The primary focus of most of the studies cited above has been the transfer of mobile species (electrons, ions and solvent), since these are the most directly linked to the measured responses, for example current-voltage curves. However, partition into and transport through the film of the mobile species is clearly highly dependent on the characteristics of the medium through which they move. One obvious example is that the rate of ion diffusion through a fluid-like film will be dependent upon the film viscosity. As will be discussed below, the latter can be related to film viscoelastic properties, which are ultimately a manifestation of polymer chain dynamics. Through arguments of this nature, it becomes clear that polymer dynamics (film viscoelastic properties) can generally be expected to be key determinants of device performance in practical applications.

We have recently described the evolution of polyaniline viscoelastic properties during film growth from aqueous perchloric acid solution [18]. However, there are a number of studies which indicate that the structure and properties of polyaniline films show significant dependence on the background electrolyte composition, notably concentration and the identity of the "dopant" anion $[16,19]$. Based on SEM images $[3,16]$ and electrochemical quartz crystal microbalance (EQCM) data $[3,10,16]$ of polyaniline 
films, it has been proposed that anions fall into two categories. Empirically, so-called "class 1" anions $\left(\mathrm{BF}_{4}^{-}, \mathrm{ClO}_{4}{ }^{-}\right.$and $\left.\mathrm{CF}_{3} \mathrm{CO}_{2}{ }^{-}\right)$lead to compact films, while so-called "class 2 " anions $\left(\mathrm{SO}_{4}{ }^{2-}, \mathrm{NO}_{3}{ }^{-}\right.$and $\left.\mathrm{Cl}^{-}\right)$lead to more open films [16]; however, the underlying rationale for this distinction is not clear. Correlation of EQCM and SEM morphological data could in principle be helpful in this respect, but the fact that the former technique is applied in situ and the latter ex situ means that comparisons are not necessarily straightforward. Simplistically, one might reasonably speculate that the void spaces will be filled with solvent in situ. Since the amount of void space is observed to be quite different for polyaniline prepared using anions from different "classes", the hypothesis we explore here is that the different film solvent populations will result in varying degrees of plasticisation and thereby different film viscoelastic properties.

The experimental approach is to use a high frequency acoustic wave device (a thickness shear mode, TSM, resonator [20]) to determine the surface mechanical impedance, from which one can extract the film mechanical behaviour [21]. We parameterize this in terms of the shear modulus $(\mathbf{G})$, expressed as the complex quantity $G^{\prime}+j G^{\prime \prime}$, where $G^{\prime}$ is the storage modulus, $\mathrm{G}^{\prime \prime}$ is the loss modulus and $\mathrm{j}$ indicates the phase relationship. The three objectives we address in this report are the influences (if any) on film shear modulus of (i) anion identity during polyaniline electrodeposition, (ii) applied potential (film charge state) during subsequent redox cycling in background electrolyte, and (iii) changing anion between deposition and redox cycling. Answers to these questions should be relevant to the preparation and exploitation of polyaniline films in practical applications.

\section{EXPERIMENTAL}

\subsection{Instrumentation}

The EQCM and crystal impedance instrumentation have been described in detail elsewhere [22-24]. For both film deposition and subsequent characterization experiments, a conventional three electrode cell was used. Polished AT-cut $10 \mathrm{MHz}$ quartz crystals (International Crystal Manufacturing Co., Oklahoma City, USA) with Au electrodes (electrochemical and piezoelectric active areas, respectively, of 0.23 and $0.21 \mathrm{~cm}^{2}$ ) were 
used. The crystal was mounted at the bottom of the cell using silicone sealant (DowCorning $3145 \mathrm{RTV}$ ) so that one of the Au electrodes was exposed to the solution and acted as the working electrode. The reference electrode, against which all potentials were measured and are reported, was a home-constructed saturated calomel electrode (SCE). The counter electrode was a Pt gauze.

Crystal admittance spectra were acquired using a Hewlett Packard HP8751A network analyzer, connected to a HP87512A transmission/reflection unit via a $50 \Omega$ coaxial cable $[14,25,26]$. Electrochemical and crystal admittance spectral data collected via a computer running HPVEE software and connected to the network analyzer through an HP10833B interface card. The surface mechanical impedance, $Z_{s}$, of the acoustic wave resonator was obtained by measuring the scattering parameter, $\mathrm{S}_{11}(\mathrm{f})$, of the transmission/reflection unit as a function of frequency, $f$, in the vicinity of the crystal resonance $[25,26]$ :

$$
Z_{s}(f)=Z_{0}\left(\frac{1+S_{11}(f)}{1-S_{11}(f)}\right)
$$

where $Z_{0}$ is the standard impedance of the system (here, $50 \Omega$ ). For calibration purposes, initial measurements were carried out on the bare crystal in air and in solution (both before film deposition) to obtain crystal and solution parameters, respectively $[18,27]$.

\subsection{Materials and procedures}

Aniline (Alfa Aesar, 99\%), $\mathrm{HClO}_{4}(\mathrm{BDH}), \mathrm{H}_{2} \mathrm{SO}_{4}$ (Aldrich) and $\mathrm{HCl}$ (Aldrich) were used as received. Polyaniline films were grown potentiodynamically $(-0.2 \leq \mathrm{E} / \mathrm{V} \leq 0.9 ; \mathrm{v}=10$ $\mathrm{mV} \mathrm{s}^{-1}$ ) from aqueous $0.1 \mathrm{M}$ aniline / $1 \mathrm{M}$ aqueous acid $\left(\mathrm{HClO}_{4}, \mathrm{H}_{2} \mathrm{SO}_{4}\right.$ or $\left.\mathrm{HCl}\right)$. A fresh sample of solution was used for each deposition experiment. All deposition and characterization measurements were carried out at room temperature $\left(20 \pm 2{ }^{\circ} \mathrm{C}\right)$.

During deposition, acoustic admittance spectra were recorded at $2 \mathrm{~s}(20 \mathrm{mV})$ intervals. Preliminary experiments showed that the rate of film deposition is different for the 
different deposition media. However, we wished to determine materials properties that may differ in different thickness regimes $[3,10,16,28-30]$. Consequently, we terminated the deposition experiments after different numbers of potential cycles such that the resultant films were of comparable thickness (i.e. similar polymer coverage). Typically, this was after 20 cycles in $\mathrm{HClO}_{4}, 10$ cycles in $\mathrm{HCl}$ and 9 cycles in $\mathrm{H}_{2} \mathrm{SO}_{4}$, at which point the cell was disconnected at the negative end of a potential cycle.

Practically, one can extract accurate shear modulus data from admittance spectra where there is sufficient damping $(>10 \%$ drop in peak admittance from that for the bare electrode) to distinguish it from the acoustically thin regime, but not so much that the signal:noise ratio is compromised (> 80\% drop in peak admittance) [18]. The conditions above satisfied this requirement, a fact that was readily observed visually on screen as the raw spectra were acquired. Expressed in more fundamental terms, this corresponds to an acoustic phase shift $(\varphi)$ greater than $20^{\circ}$ [18] (below which the film is regarded as acoustically "thin") but less than $90^{\circ}$ (where complications associated with film resonance arise [31-33]). The value of $\varphi$ (for a shear acoustic wave of angular frequency, $\omega=2 \pi \mathrm{f}$ ) across a film of thickness $\mathrm{h}_{f}$ and density $\rho_{f}$ is related to the viscoelastic properties of the film by [24, 27]:

$$
\varphi=\gamma h_{f}=\omega h_{f} \sqrt{\rho_{f}} \sqrt{\frac{1+G^{\prime}|| G \mid}{2|G|}}
$$

where:

$$
\sqrt{G}=\left(\frac{|G|+G^{\prime}}{2}\right)^{1 / 2}+j\left(\frac{|G|+G^{\prime}}{2}\right)^{1 / 2}
$$

and

$$
|G|=\left[\left(G^{\prime}\right)^{2}+\left(G^{\prime \prime}\right)^{2}\right]^{1 / 2}
$$


Following deposition, the film was rinsed with distilled water and transferred to fresh monomer-free background electrolyte for voltammetric and acoustic characterization (in the range $-0.2<\mathrm{E} / \mathrm{V}<0.5$, spanning the first redox process). Two types of experiment were carried out, one in which the film was characterized using the same acid (anion) solution as for deposition and one in which the acid (anion) was changed.

\subsection{Data Fitting}

Procedures for the extraction of shear moduli (storage and loss components) have been discussed in detail elsewhere $[18,22,24,27,34]$. Values of $\mathrm{G}^{\prime}$ and $\mathrm{G}^{\prime \prime}$ were calculated using the Maple package by fitting experimental impedance (Z) data using [21, 22, 34, 35]:

$$
Z=j \omega \rho_{s}+Z_{f}\left[\frac{Z_{L} \cosh \left(\gamma h_{f}\right)+Z_{f} \sinh \left(\gamma h_{f}\right)}{Z_{f} \cosh \left(\gamma h_{f}\right)+Z_{L} \sinh \left(\gamma h_{f}\right)}\right]
$$

In Eq. $5, Z_{f}$ is the characteristic mechanical impedance of the film:

$$
Z_{f}=\left(\rho_{f} G\right)^{1 / 2}
$$

$\gamma$ is the shear wave propagation constant in the film $[21,25,27]$ :

$$
\gamma=j \omega / v_{f}=j \omega\left(\rho_{f} / G\right)^{1 / 2}
$$

and $\mathrm{Z}_{\mathrm{L}}$ is the characteristic mechanical impedance of the solution (a semi-infinite Newtonian fluid) $[18,27]$ :

$$
Z_{L}=\left(\frac{\omega \rho_{L} \eta_{L}}{2}\right)^{1 / 2}(1+j)
$$


$\rho_{\mathrm{L}}$ and $\eta_{\mathrm{L}}$ are the density and the viscosity of the solution, respectively, and $\mathrm{v}_{\mathrm{f}}$ is the acoustic wave velocity across the film.

Polymer trapped within surface features (roughness) of the electrode was treated as a rigidly coupled ideal mass and is represented by the first term on the right hand side of Eq. 5. To determine viscoelastic properties, one should minimize the effect of this term with respect to the second (viscoelastic) term in Eq. 5. This was accomplished by use of polished crystals, for which the scale of roughness (ca. $5 \mathrm{~nm}$ by AFM imaging) is ca. 1$5 \%$ of the film thickness across the range where shear moduli are determined [18].

In principle, in order to fit the shear moduli, one requires values of film density, $\rho_{f}$, and thickness, $\mathrm{h}_{f}[18,33]$, although we note that fitted values of shear moduli for soft films show a general insensitivity to the film density value. In the specific case of polyaniline exposed to aqueous solution, the situation is simplified further, due to the similar densities of aniline (monomer density, $\left.\rho_{M}=1.02 \mathrm{~g} \mathrm{~cm}^{-3}\right)$ and water $\left(\rho_{\mathrm{S}}=1.0 \mathrm{~g} \mathrm{~cm}^{-3}\right)$. A film density of $\rho_{f}=1 \mathrm{~g} \mathrm{~cm}^{-3}$ was used, but any ambiguity about film solvation introduces negligible uncertainty into the film density used to fit the shear moduli. As described previously [18], values of film thickness were obtained by an optimization process, that combines a coulometric assay (from molar coverage, $\Gamma_{f}$, and monomer density, $\rho_{M}$ ) to generate the physically allowable lower boundary of $h_{f}$ with the requirement for real and positive values of both $\mathrm{G}^{\prime}$ and $\mathrm{G}^{\prime \prime}$. Together, these constraints define a narrow range of permissible film thickness, within which $G^{\prime}$ and $G^{\prime \prime}$ values varied very little. As a further check, at the end of selected experiments, AFM images were taken to assess film thickness directly. The outcomes were consistent with the acoustically determined values and also revealed some roughness, which the acoustic experiment "averages" across the crystal.

As a final test of internal consistency, shear moduli were used to calculate the acoustic wave decay length in the propagation direction, perpendicular to the interface $[18,25$, 26]: 


$$
\delta=1 / \gamma=\frac{1}{\omega \sqrt{\rho_{f}}} \sqrt{\frac{2|G|}{1-G^{\prime} /|G|}}
$$

One would expect to be able to obtain physically realistic and accurate values of shear modulus components when $0.1 \leq \mathrm{h}_{f} / \delta \leq 2$ [18]. The lower bound marks the point at which viscoelastic effects cease to be significant. At the upper bound, the round trip for the acoustic wave (to the outer film interface, reflection, and back to the electrode) is $4 \delta$, at which point the intensity of the reflected wave is less than $2 \%$ of that for the outgoing wave, so signal quality becomes limiting.

\section{Results and Discussion}

\subsection{Film deposition in the presence of different anions}

\subsubsection{Qualitative overview of raw data}

Fig. 1 shows the raw voltammetric and acoustic data for polyaniline deposition in the presence of each of the different anions. Data were collected during all deposition cycles, continuously in the case of the current and at $20 \mathrm{mV}$ intervals in the case of the admittance spectrum. However, for presentational clarity, only selected responses are shown. Panels $a-c$ show the i-E curves in the presence of $\mathrm{SO}_{4}{ }^{2-}, \mathrm{Cl}^{-}$and $\mathrm{ClO}_{4}{ }^{-}$anion, respectively; these were reproducible and similar to those reported in the literature $[3,4$, 29, 30, 36]. Corresponding admittance spectra, acquired at the end of the indicated deposition scans (at $\mathrm{E}=-0.2 \mathrm{~V}$ ) are shown in panels $d-f$. Note that, since the patterns of admittance and current variation with anion are different, we show data for different numbers of deposition cycles in panels $a-c$ and $d-f$. Without imposition of any model, these variations immediately signal the formation of materials with distinct properties, which we now characterize.

The obvious qualitative trend in peak admittance values seen in panels $d-f$ is progressive damping with film growth. Consider first the early stages of polymerization. In panel $d$ ( $\mathrm{SO}_{4}{ }^{2-}$ anion), the peak admittance after a single deposition cycle is decreased to ca. $80 \%$ of that for the bare electrode in the deposition solution. Similar increases in damping in $\mathrm{Cl}^{-}$and $\mathrm{ClO}_{4}^{-}$media (panels $e$ and $f$ ) require 2-3 and 7 deposition cycles, respectively. 
Turning to the end of the deposition experiments shown (9, 10 and 19 cycles) for $\mathrm{SO}_{4}{ }^{2-}$, $\mathrm{Cl}^{-}$and $\mathrm{ClO}_{4}{ }^{-}$media, respectively, the resonant admittance had diminished to 13, 22 and $40 \%$ of that for the bare electrode. In other words, the experiment with the least number of deposition cycles $\left(\mathrm{SO}_{4}{ }^{2-}\right)$ showed the largest damping effects and the experiment with the most number of deposition cycles $\left(\mathrm{ClO}_{4}^{-}\right)$showed the least damping.

It is clear that the type of anion present during polyaniline deposition exerts a decisive influence on film rheological properties. The goal now is to distinguish the contributions to this from the rate of film growth and the intrinsic materials properties, both of which vary across the portfolio of experiments represented in Fig. 1.

The data of Fig. 1 show that damping (energy loss) increases most rapidly (with respect to the number of deposition cycles) fastest for $\mathrm{SO}_{4}{ }^{2-}$ and slowest for $\mathrm{ClO}_{4}{ }^{-}$. At a superficial level, one might interpret this to mean that polyaniline films are progressively softer in the order $\mathrm{ClO}_{4}{ }^{-}>\mathrm{Cl}^{-}>\mathrm{SO}_{4}{ }^{2-}$. However, different growth rates in these three media mean that the loss (damping) relates to different amounts of polymer and is thus not itself a material property. This variation of growth rate with anion has been reported by a number of other authors $[3,16,29,30,37-40]$ and the consensus view is that the relative growth rates in aqueous acid media are in the order $\mathrm{H}_{2} \mathrm{SO}_{4}>\mathrm{HCl}>\mathrm{HClO}_{4}$. Consistent with this, in the experiments of Fig. 1 (panels $a-c$ ) the polymerization currents in $\mathrm{Cl}^{-}$and $\mathrm{ClO}_{4}{ }_{4}^{-}$, respectively, are ca. $25 \%$ and $2.5 \%$ of that in $\mathrm{SO}_{4}{ }^{2-}$. Various authors have rationalised this in terms of: (i) different polymer growth mechanisms [40-42] (involving progressive or instantaneous nucleation, 2D or 3D growth and the presence of diffusional control, according to the electrolyte); (ii) stability differences (due to differing anion lyophilicity $[3,40])$ in ion pair formation between the anion and the anilinium species produced during monomer oxidation; or (iii) the Gibbs energy change associated with ion formation in aqueous solution [30], which follows the order $\mathrm{SO}_{4}{ }^{2-}<\mathrm{Cl}^{-}<\mathrm{NO}_{3}{ }^{-}<$ $\mathrm{ClO}_{4}{ }^{-}$for polyaniline salt formation. We do not duplicate these efforts by pursuing the origins of these effects, but rather extend the investigation by exploring their consequences in terms of materials properties. In this respect, we note two examples of the qualitative recognition of a relationship between growth rate and properties. First, 
faster polyaniline growth (by a factor of 3) in $\mathrm{SO}_{4}{ }^{2-}$ than in $\mathrm{ClO}_{4}{ }^{-}$was attributed $[7,43]$ to a more open morphology in $\mathrm{SO}_{4}{ }^{2-}$ and a more compact morphology in $\mathrm{ClO}_{4}{ }^{-}$. Second, direct observation of surface features by SEM images supported the same order of polyaniline film growth (fastest in $\mathrm{SO}_{4}{ }^{2-}$, moderate in $\mathrm{Cl}^{-}$and slowest in $\mathrm{ClO}_{4}{ }^{-}$) [3] .

\subsubsection{Coulometric and Acoustic Responses of Film Deposition}

Fig. 2 shows the coulometric (Q; panel $a)$, and the acoustic $(\Delta \mathrm{f}$; panel $b$ ) responses as functions of time $(t / s)$ and their direct correlation ( $\Delta \mathrm{f}-\mathrm{Q}$ plot; panel $c)$. Each panel shows three data sets representing deposition in the presence of each of the anions $\left(\mathrm{SO}_{4}{ }^{2-}, \mathrm{Cl}^{-}\right.$, and $\mathrm{ClO}_{4}{ }^{-}$, as indicated). The responses in panels $a$ and $b$ all contain two components, an oscillatory one and a monotonic one. The oscillatory components represent changes within a deposition cycle and are attributable to the reversible redox switching of film deposited in previous cycles. This is shown in more detail for a representative cycle in the insets in panels $a$ and $b$. The magnitude of this component progressively increases during the experiment, as polyaniline is deposited. The monotonically increasing component is attributable to the irreversible polymerisation and deposition of the film. Interestingly, this component is initially linear, but after a few cycles (typically three in $\mathrm{SO}_{4}{ }^{2-}$ and $\mathrm{Cl}^{-}$ media and seven in $\mathrm{ClO}_{4}^{-}$) increases more sharply with time. This behaviour has been attributed previously to self-catalytic behaviour [16, 44-46] and/or the increase in surface area associated with a rough film [47].

A similar explanation applies to the frequency response data (panel $b$ ), with regard to the presence of oscillatory and monotonically increasing components. Here, differences in film growth rates in the different electrolytes result in resonant frequency responses (representative of surface coverage, initially gravimetrically and subsequently in a more complex manner) with a time dependence that is linear in $\mathrm{ClO}_{4}^{-}$, almost linear in $\mathrm{Cl}^{-}$ (though with a gradient ca. three times greater than in $\mathrm{ClO}_{4}{ }^{-}$) and nonlinear (power law significantly greater than unity) in $\mathrm{SO}_{4}{ }^{2-}$.

Direct correlation between the change in resonant frequency and charge is shown in panel $c$ of Fig. 2. The initial stages of the film deposition - regardless of the nature of 
background electrolyte - are characterised by a linear $\Delta \mathrm{f} v s \mathrm{Q}$ relationship. This corresponds to the acoustically thin regime in which the frequency response is interpretable gravimetrically. Surprisingly, in this regime (labelled "acoustically thin regime" in Fig. 2 panel c) the deposition data for the different electrolytes virtually superimpose. At this point it is worth noting that the charge response represents the consumption of monomer and the frequency response represents the formation and deposition on the electrode surface of polymer. Within the acoustically thin regime, the relationship between the two can, in principle, be influenced by Faradaic efficiency, as well as solvent content; as one moves into the acoustically thick regime, viscoelastic effects also come into play. Staying with the acoustically thin regime for the present, combination of the Sauerbrey equation (for the frequency response) with Faraday's law (for the coulometric response) yields the equivalent molar mass of deposited polymer, $M$ $/ \mathrm{g} \mathrm{mol}^{-1}$ :

$$
M=\frac{n F A_{e}}{Q} \frac{\Delta f}{C_{1}}
$$

where $\mathrm{C}_{1}=-2.26 \times 10^{8} \mathrm{~Hz} \mathrm{~cm}^{2} \mathrm{~g}^{-1}$ represents the combination of quartz crystal constants and the other symbols have their usual meanings [18]. Applying Eq. 10 to the acoustically thin regime data at $\mathrm{E}=-0.2 \mathrm{~V}$ (negative extremes of potential cycles) of Fig. 2 panel $c$, one obtains $M=93.2 \mathrm{~g} \mathrm{~mol}^{-1}$. This is remarkably close to the molar mass of a monomeric aniline unit, suggesting that thin reduced films contains relatively little solvent; the nominal absence of dopant from these films in the reduced state means that the anion contribution to the mass signal is also small.

At higher deposition charges (longer times), the frequency response (see Fig. 2, panel c) departs from the limiting linear relationship found at lower charge. This is a clear indication of regime change from rigid to viscoelastic. The manner in which this occurs varies with anion identity. The $\Delta \mathrm{f}-\mathrm{Q}$ relationship for films grown in $\mathrm{ClO}_{4}{ }^{-}$and $\mathrm{SO}_{4}{ }^{2-}$ follow the same trajectory, although at different rates: the rate of change of resonant frequency is much higher in $\mathrm{SO}_{4}{ }^{2-}(18 \mathrm{kHz}$ in 9 deposition cycles $)$ than in $\mathrm{ClO}_{4}{ }^{-}(13 \mathrm{kHz}$ 
in 20 deposition cycles). As a crude comparison, the average rate of traversing the plot is ca. $2 \mathrm{kHz}$ per deposition cycle from $\mathrm{SO}_{4}{ }^{2-}$ medium, $1.3 \mathrm{kHz}$ per cycle from $\mathrm{Cl}^{-}$and $<1$ $\mathrm{kHz}$ per cycle from $\mathrm{ClO}_{4}^{-}$.

\subsubsection{Effect of anion on film viscoelastic properties during deposition}

In this section, we explore the temporal evolution of film mechanical properties during deposition as a function of the anion present, via the shear modulus component values as functions of anion and film thickness. This explicitly focuses on the direct effect of the medium of preparation on the resultant film properties. In a subsequent section, we will focus on if/how exposure to a different medium re-directs the film dynamics, which will test the film's "memory" of preparation conditions.

Fig. 3 shows values of $\mathrm{G}^{\prime}$ and $\mathrm{G}^{\prime \prime}$ as a function of the growing thickness of polyaniline films during deposition from different electrolytes. The final values of film thickness for the films grown from $\mathrm{HClO}_{4}, \mathrm{HCl}$ and $\mathrm{H}_{2} \mathrm{SO}_{4}$ were ca. $0.6,0.5$ and $0.7 \mu \mathrm{m}$, respectively. This careful use of control parameters to generate films of similar thickness (irrespective of the deposition rate, see above) allows fair comparisons to be made of film mechanical properties within the same thickness regime. AFM images of the films revealed some surface roughness (typically, film thicknesses are mean values with a vertical feature size of ca. $50 \mathrm{~nm}$, i.e. $\sim 0.1 \mathrm{~h}_{\mathrm{f}}$ ), so the shear modulus values presented here should be regarded as effective averages; since the principle of this point has already been addressed in some detail for PEDOT films [48] we do not dwell on it further here.

For all electrolytes, both shear modulus components increase with film thickness, but to an extent that varies with respect to the anion. $\mathrm{G}^{\prime}$ values at any given thickness decrease with anion identity in the order $\mathrm{ClO}_{4}{ }^{-}>\mathrm{SO}_{4}{ }^{2-}>\mathrm{Cl}^{-}$. At the end of the deposition process, for the films represented by the experiments of Fig. 1, $\mathrm{G}^{\prime}$ values for the reduced films (E $=-0.2 \mathrm{~V})$ were $1.8 \times 10^{7}\left(\mathrm{ClO}_{4}{ }^{-}\right), 1.5 \times 10^{7}\left(\mathrm{SO}_{4}{ }^{2-}\right)$ and $9.2 \times 10^{6}\left(\mathrm{Cl}^{-}\right)$dyn $\mathrm{cm}^{-2}$. One can express the extent of variation of storage modulus with respect to the growing film thickness by comparing the value after the final deposition cycle, $\mathrm{G}_{\mathrm{f}}^{\prime}$, with that after the first cycle at which it can be evaluated, $\mathrm{G}_{i}^{\prime}$; in practice, $\mathrm{G}_{i}^{\prime}$ corresponds to the value at $\mathrm{h}_{\mathrm{f}}$ 
$\sim 0.1 \mu \mathrm{m}$. To facilitate comparison of films in different media, with different absolute values of shear modulus, we define a normalized quantity, $\psi^{\prime}=\left(G_{f}^{\prime}-G_{i}^{\prime}\right) / G_{f}^{\prime}$. We find the increase in $\mathrm{G}^{\prime}$ values of polyaniline films grown in $\mathrm{HClO}_{4}$ corresponds to $\psi^{\prime}=0.8$, experimentally indistinguishable from that value for films grown in $\mathrm{HCl}$, and slightly larger than that for films grown in $\mathrm{H}_{2} \mathrm{SO}_{4}$, for which $\psi^{\prime}=0.7$.

The loss modulus, $\mathrm{G}^{\prime \prime}$ (see Fig. 3, panel $b$ ), data show similar trends as do the $\mathrm{G}^{\prime}$ data with regard to anion, and the final $\mathrm{G}^{\prime \prime}$ values in the different media are broadly similar: $2 \times 10^{7}\left(\mathrm{ClO}_{4}^{-}\right), 1.13 \times 10^{7}\left(\mathrm{SO}_{4}{ }^{2-}\right)$ and $7.9 \times 10^{6}\left(\mathrm{Cl}^{-}\right) \mathrm{dyn} \mathrm{cm}^{-2}$. The main difference was the somewhat greater variation (as compared to $\mathrm{G}^{\prime}$ ) during deposition. Similarly, we define $\psi^{\prime \prime}=\left(G_{f}^{\prime \prime}-G_{i}^{\prime \prime}\right) / G_{f}^{\prime \prime}$. We find that $\psi^{\prime \prime}=0.97,0.94$ and 0.92 for films grown in $\mathrm{ClO}_{4}^{-}$, $\mathrm{SO}_{4}{ }^{2-}$ and $\mathrm{Cl}^{-}$, respectively. Given the likely experimental uncertainties, we view these relative values as not significantly different, although of course the absolute values of $\mathrm{G}^{\prime \prime}$ are significantly different. Based on the magnitude of $\mathrm{G}^{\prime \prime}$ at short times (ca. $6.7 \times 10^{5} \mathrm{dyn}$ $\mathrm{cm}^{-2}$ for all films), we suggest that the film is rather liquid-like, i.e. a layer of diffuse solvent-separated polymer strands. By the end of deposition, in-filling between the polymer strands (replacement of solvent by polymer) results in composition and properties more typical of a bulk viscoelastic material, in particular loss tangents of 1.0 $\left(\mathrm{ClO}_{4}{ }^{-}\right), 0.8\left(\mathrm{SO}_{4}{ }^{2-}\right)$ and $0.86\left(\mathrm{Cl}^{-}\right)$, respectively. Interestingly, the shear modulus components in $\mathrm{SO}_{4}{ }^{2-}$ and $\mathrm{Cl}^{-}$follow the same trajectory with film thickness. This similarity of film characteristics is consistent with the grouping of these dopants within the same family (so-called "class 2" anions). Furthermore, the lower shear moduli (cf. $\left.\mathrm{ClO}_{4}{ }^{-}\right)$provides a link between their electrochemical dynamics and morphology $[3,16]$ : more open structures are associated with greater solvation, which facilitates chain motion and thereby generates a medium of lower viscosity through which dopant ions can diffuse more rapidly.

From the shear moduli shown in Fig. 3, we can determine the decay length (defined by Eq. 9) and the acoustic phase shift ( $\varphi$, defined by Eq. 2); the outcomes are shown in Fig. 4. We note that $\delta$ is a material property, but $\varphi$ is a property of both the material and the 
sample (via film thickness). The decay lengths all follow a similar pattern and a combination of the relatively similar shear moduli and their modest (square root) influence over decay length results in similar long time limiting values of decay length in all media, ca. $2 \mu \mathrm{m}$. It is worth noting that the decay length is always significantly larger than the film thickness $\left(h_{f} \leq 0.7 \mu \mathrm{m}\right)$. This validates the "acoustically thick but finite film" analysis used here; the system never enters the semi-infinite regime, in which the response becomes insensitive to film thickness because the acoustic wave does not traverse the film.

In contrast to the decay length, the acoustic phase shift (Fig. 4, panel $b$ ) steadily increases with the film thickness. Over the interval shown, the phase shift for each film remains in the optimal range for shear modulus determination. In particular, it does not approach the value of $\pi / 2$ associated with film resonance. Again, the modelling protocol is validated. The phase shift trajectories of films grown in $\mathrm{H}_{2} \mathrm{SO}_{4}$ and $\mathrm{HCl}$ are similar to each other, while the phase shift trajectory of films grown in $\mathrm{HClO}_{4}$ is distinct and traverses the phase shift dimension more slowly. This is another manifestation of the fact that films grown in $\mathrm{HClO}_{4}$ are stiffer (less deformable) than those grown in either $\mathrm{H}_{2} \mathrm{SO}_{4}$ or $\mathrm{HCl}$.

\subsection{Polyaniline film characterisation in monomer-free electrolytes}

Having discussed the viscoelastic signatures of polyaniline films as a function of thickness during growth from different media, we now shift to determination of shear moduli in monomer-free electrolyte solutions. The obvious variation we explore is that with film oxidation state (doping level), as controlled via applied potential. We addressed this for two parallel sets of experiments: (i) for films exposed to the same electrolyte (anion) as used in film deposition and (ii) for films exposed to a different anion to that used in film deposition. In the latter case, we illustrate the principles by showing data for one representative permutation of deposition / cycling anions, rather than presenting an encyclopaedic tour of all possible permutations.

Fig. 5 shows cyclic voltammograms of polyaniline films recorded in the same monomerfree background electrolytes as those used for film deposition. Peak shapes, currents and 
potentials for film oxidation vary with the type of anion. Films grown and cycled in $\mathrm{HClO}_{4}$ show sharper oxidation peaks and higher $\mathrm{I}_{\mathrm{pa}} / \mathrm{I}_{\mathrm{pc}}$ values than for the other two electrolytes used: $\mathrm{I}_{\mathrm{p} a} / \mathrm{I}_{\mathrm{p}}=3,2$ and 1.6 for $\mathrm{HClO}_{4}, \mathrm{H}_{2} \mathrm{SO}_{4}$ and $\mathrm{HCl}$, respectively. In contrast, the peak potentials of films grown and cycled in all three electrolytes are very similar. While the emphasis of the present study is on polymer dynamics, we note that electron dynamics - which underlie the i-E response here - have been explored by other workers. In particular, ESR [29] and in-situ conductivity [37] experiments revealed that the use of $\mathrm{ClO}_{4}{ }^{-}$as a dopant yields more metal-like polyaniline films than does use of $\mathrm{SO}_{4}{ }^{2-}$. This was attributed to greater interaction of $\mathrm{SO}_{4}{ }^{2-}$ ions with the positive charges (polarons) on the polymer chains and thereby greater charge localisation. We suggest that in general polymer-anion interactions can be expected to constrain polymer chain dynamics and thus be a determinant of film mechanical properties.

In Fig. $6, \mathrm{G}^{\prime}$ and $\mathrm{G}^{\prime \prime}$ values are shown for polyaniline films exposed to various background electrolyte solutions and subjected to a cyclic linear potential sweep. The qualitative potential dependences of shear moduli values (both storage and loss components) are similar in all background electrolytes used. Starting from a fully reduced film and scanning to positive potentials, both shear modulus components are constant for $\mathrm{E}<0.15 \mathrm{~V}$ then increase monotonically throughout the first redox process $(0.15<\mathrm{E} / \mathrm{V}<$ $0.5)$. In the reduction half cycle, the trends are reversed. There is clear hysteresis with respect to scan direction in all data sets, but this is more pronounced for films grown and characterized in $\mathrm{SO}_{4}{ }^{2-}$ than for those grown and characterized either in $\mathrm{ClO}_{4}{ }^{-}$or $\mathrm{Cl}^{-}$; the same is true for films deposited in $\mathrm{Cl}^{-}$then subsequently cycled in $\mathrm{SO}_{4}{ }^{2-}$.

Quantitatively, the shear modulus values for different anion exposure regimes are distinctly - though not dramatically - different. For films deposited and cycled in the same electrolyte, the shear moduli (both components) decrease in the order $\mathrm{ClO}_{4}{ }^{-}>\mathrm{SO}_{4}{ }^{2-}$ $>\mathrm{Cl}^{-}$. The interesting follow-up question is whether the film retains a "memory" of the deposition conditions, for example via a templating or other structural feature of the anion/polymer combination, or simply adopts a structure and dynamics that correspond to the immediate ion of exposure. The evidence we found, as illustrated in Fig. 6 by the 
example of a film grown in $\mathrm{Cl}^{-}$then cycled in $\mathrm{SO}_{4}{ }^{2-}$, is that there is a clear response to changing anion identity. For this particular example, the observed shifts of the $\mathrm{G}^{\prime}$ and $\mathrm{G}^{\prime \prime}$ values towards those of the "new" electrolyte - indistinguishably so for G" - were complete within one potential cycle.

Analogous effects have been reported [28] in photocurrent transient experiments, in which photocurrents produced by polyaniline films were sensitive to the particular electrolyte solution (probing medium) rather than to the deposition solution (preparation medium). The same has also been observed for electrochemical impedance responses [49], which are governed by a combination of electron and ion dynamics. Turning to the polymer component of the system, SEM images of newly prepared films show film morphologies that are characteristically different for different deposition medium anions $[3,16]$; the effect of cycling in changed electrolytes was not explored using this technique. In our experiments, we attribute this rather significant property of responding to the electrolyte to the fact that the films are relatively soft, so the polymer chains are mobile (on the timescale of the experiment) and can reconfigure their molecular geometry to that optimum for the electrolyte that permeates them at any given time.

The relatively modest, though indisputable, variations of $\mathrm{G}^{\prime}$ and $\mathrm{G}^{\prime \prime}$ with potential are highlighted by means of a differential presentation format in Fig. 7. We have previously shown (for the restricted case of a single electrolyte) that it is useful to define normalized differential (with respect to potential) functions of the shear modulus components as follows:

$$
\xi^{\prime}=\frac{1}{G_{(-0.2 V)}^{\prime}} \frac{d G^{\prime}}{d E}
$$

and

$$
\xi^{\prime \prime}=\frac{1}{G_{(-0.2 V)}^{\prime \prime}} \frac{d G^{\prime \prime}}{d E}
$$


Through the magnitudes of $\xi^{\prime}$ and $\xi^{\prime \prime}$ we see that, in general, relative variations of G" with potential are larger than those of $\mathrm{G}^{\prime}$. With respect to anion, the variations of $\xi^{\prime}(\mathrm{E})$ are perhaps slightly larger in $\mathrm{ClO}_{4}^{-}$, particularly during reduction, but the effect is not large. On the other hand, the variations of $\xi^{\prime \prime}(\mathrm{E})$ are significantly larger in $\mathrm{SO}_{4}{ }^{2-}$ than in $\mathrm{ClO}_{4}{ }^{-}$or $\mathrm{Cl}^{-}$. One feature that is noticeable is that the marked asymmetry with respect to potential scan direction that is visible in the i-E responses (and widely reported in the polyaniline literature) is not seen in the $\xi^{\prime}$-E and $\xi^{\prime \prime}$-E responses. The implication is that the factors that determine these responses are distinct in some way: since charged species (electron/ion) populations and motions control the current response, it is a reasonable speculation that uncharged (e.g. solvent) species populations are more directly correlated with viscoelastic properties.

Correlating our observations more widely with those in the literature (see above), the picture is one in which probes of electron, ion and polymer dynamics all show film response to the immediate electrolyte of exposure; there is no permanent "memory" of the deposition medium. We suggest that there are three significant features of the polymer-based dynamics explored here through viscoelastic properties. First, one of the factors in controlling electron mobility will be chain planarity, which in turn will be partly controlled by polymer/anion interactions. The ability of the polymer chains to move and re-orient to the structure - of which ring-ring co-planarity is one aspect associated with a given anion thus influences the facility for electron transport rates to respond to electrolyte composition. Second, one can relate the loss modulus to the viscosity $(\eta)$ through the relationship $\mathrm{G}^{\prime \prime}=\omega \eta$, where $\omega=2 \pi f$ is the angular frequency of the modulation. Thus, changes in $\mathrm{G}^{\prime \prime}$ control the film viscosity, which in turn (simplistically through the classical Stokes-Einstein relation, $\mathrm{D} \alpha 1 / \eta$ ) governs the rate of ion diffusion; if one wishes to focus on ion migration, the classical Einstein equation relates mobility linearly to D. Finally, we note that the larger scale motions in both length and mass terms of polymer segmental motion, as compared to the motions of mobile species (electrons, ions and solvent), will generally result in polymer being the slowest to respond. The fact that we do observe changes in polymer dynamics means that the 
cascade of other processes (associated with electron, ion and solvent transport) will necessarily occur.

The one feature that we have not explored here is the relationship of polymer dynamics to film solvation. In order to do this, we require the absolute population of solvent within the film; gravimetric measurements using the EQCM on acoustically thin films only provide relative populations, i.e. population changes derived from mass (frequency) changes. In a subsequent study we will pursue this matter using neutron reflectivity, a powerful in situ technique capable of determining both the overall absolute populations of film components and their spatial distributions.

\section{CONCLUSIONS}

Using thickness shear mode resonators operating at $10 \mathrm{MHz}$, we have been able to determine storage and loss shear moduli in situ for polyaniline films in the thickness range 0.1-0.7 $\mu \mathrm{m}$ during their potentiodynamic electrochemical deposition, and subsequently during redox cycling in background electrolyte. This has been accomplished for a range of dopant anions (perchlorate, sulphate, chloride). The two general results are that the film viscoelastic properties evolve during the deposition process and that they respond significantly to the identity of the dopant anion.

Simplistic considerations of shear moduli as a function of deposition time (here, controlled by the number of voltammetric cycles) are not helpful since, as has been discussed elsewhere, the deposition rate varies in the presence of different dopant anions (all other parameters being identical). Consequently, in order to make meaningful comparisons, it is necessary to consider films of the same thickness. This has been accomplished across the thickness range $\mathrm{h}_{\mathrm{f}} \sim 0.1-0.7 \mu \mathrm{m}$. Within this range, the films under all conditions have shear moduli characteristic of typical viscoelastic materials. For $\mathrm{h}_{\mathrm{f}}<0.1 \mu \mathrm{m}$ the acoustic deformation is too small for reliable viscoelastic measurements to be made and for $h_{f}>0.7 \mu \mathrm{m}$ one approaches film resonance, where the interpretation is more complicated. 
Within the range explored, we find that the shear modulus components are largest (i.e. the films are stiffest and have the greatest loss) in perchlorate medium; the values in sulphate and chloride media are similar to each other and somewhat smaller than in perchlorate medium. It is noteworthy that a previous empirical classification of anions as polyaniline dopants placed perchlorate in one category ("class 1") that is characteristic of compact films with low transport rates, and sulphate and chloride in another category ("class 2") that is characteristic of more diffuse films with faster transport rates. This pattern is consistent with the notion that the loss modulus is a measure of film viscosity, $\eta$, via $G^{\prime \prime}=\eta \omega$ : higher loss moduli represent higher viscosity and thereby slower mobile species transport (by diffusion or migration).

In monomer-free background electrolyte solution, the film shear moduli responded to the applied potential. For all anions and both components, shear moduli increased modestly with film oxidation. This effect was chemically reversible, although there was some hysteresis with applied potential on the timescale of slow scan voltammetric experiments. Through one test case, involving chloride-prepared films subsequently exposed to sulphate, we found that redox cycling in an electrolyte involving a different anion to that used for film deposition resulted in a rapid shift in viscoelastic properties towards those of the ambient medium. For the relatively soft films involved, we associate this with rapid and complete exchange of dopant within a redox cycle.

\section{ACKNOWLEDGMENTS}

MAM thanks CARA for financial support. 


\section{FIGURE LEGENDS}

Figure 1. Data for the potentiodynamic deposition of polyaniline films. Panels $a-c$ : i-E responses. Panels $d-f$ : acoustic admittance spectra at the end of selected potential cycles (as indicated by numbers on individual spectra; asterisk indicates response for bare electrode prior to initiation of polymerization), at $\mathrm{E}=-0.2 \mathrm{~V}$; selected spectra only shown, for presentational clarity. Electrolyte anion: panels $a, d$ : sulphate; panels $b, e$ : chloride; panels $c, f$ : perchlorate. Other details as in main text.

Figure 2. Coulometric and resonant frequency responses from the experiments of Fig. 1. Panel $a$ : coulometric response as a function of time. Panel $b$ : resonant frequency shift as a function of time. Panel $c$ : frequency shift (from panel $b$ ) as a function of charge (from panel a). Annotation indicates electrolyte species. Insets in panels $a$ and $b$ show reversible components of responses within a single potential cycle.

Figure 3. Shear moduli as a function of film thickness for the experiments of Fig. 1. Panel $a$ : storage modulus data. Panel $b$ : loss modulus data. Annotation indicates electrolyte species.

Figure 4. Acoustic wave propagation parameters within growing polyaniline films as a function of film thickness, $\mathrm{h}_{\mathrm{f}}$. Panel $a$ : decay length (defined by Eq. 9); panel $b$ : phase shift (defined by Eq. 2).

Figure 5. Voltammetric responses $\left(v=10 \mathrm{mV} \mathrm{s}^{-1}\right)$ for the polyaniline films prepared by the experiments of Fig. 1 following transfer to background electrolyte. In each case, the background electrolyte (see annotations) was identical to that used in the deposition experiment. Note different current scales, reflecting different i-E waveshapes.

Figure 6. Shear moduli as functions of potential for polyaniline films exposed to background electrolyte (as annotated) and subject to voltammetric potential cycles $(v=$ $10 \mathrm{mV} \mathrm{s}^{-1}$ ). Points indicate data; lines are a guide to the eye; arrows indicate scan direction. Panel $a$ : storage modulus; panel $b$ : loss modulus. In cases marked $\mathrm{HClO}_{4}$, 
$\mathrm{H}_{2} \mathrm{SO}_{4}$ and $\mathrm{HCl}$, the background electrolyte was the same for film deposition and redox cycling. In case marked $\mathrm{HCl} / \mathrm{H}_{2} \mathrm{SO}_{4}$, the film was deposited from $\mathrm{HCl}$ electrolyte and cycled in $\mathrm{H}_{2} \mathrm{SO}_{4}$ electrolyte.

Figure 7. Variations with applied potential of normalised differential shear moduli of polyaniline films exposed to different electrolytes (as annotated). Panel $a$ : differential storage moduli, $\xi^{\prime}$ (defined by Eq. 11); panel b: differential loss moduli, $\xi^{\prime \prime}$ (defined by Eq. 12). Data from Fig. 6. Points indicate data; lines are a guide to the eye; arrows indicate scan direction. 


\section{REFERENCES}

[1] H. Xue, C. Li, Z. Shen, Biosen. Bioelectron. 20 (2005) 2330

[2] I. Jureviciute, A. Malinauskas, K. Brazdziuviene, L. Bernotaite, B. Slakus, Sens. Actuators B Chem. 107 (2005) 716

[3] S.-J. Choi, S.-M. Park, J. Electrochem. Soc. 149 (2002) E26

[4] A. Baba, S. Tian, F. Stefani, C. Xia, Z. Wang, R. C. Advincula, D. Johannsmann, W. Knoll, J. Electroanal. Chem. 562 (2004) 95

[5] A. G. MacDiarmid, Synth. Met. 84 (1997) 27

[6] A. G. MacDiarmid, A. J. Epstein, Farad. Discuss. Chem. Soc. 88 (1989) 317

[7] A. A. Syed, M. K. Dinesan, Talanta 38 (1991) 815

[8] M. C. Miras, C. Barbero, R. Kotz, O. Haas, J. Electroanal. Chem. 369 (1994) 193

[9] M. C. Bernard, A. H.-L. Goff, Electrochim. Acta. 52 (2006) 595

[10] S. Pruneanu, E. Csahok, V. Kertesz, G. Inzelt, Electrochim. Acta. 43 (1998) 2305

[11] C. Gabrielli, M. Keddam, N. Nadi, H. Perrot, J. Electroanal. Chem. 485 (2000) 101

[12] D. Orata, D. A. Buttry, J. Am. Chem. Soc. 109 (1987) 3574

[13] B. Keita, A. Mahmoud, L. Nadjo, J. Electroanal. Chem. 386 (1995) 245

[14] A. R. Hillman, M. A. Mohamoud, Electrochim. Acta. 51 (2006) 6018

[15] H. N. Dinh, J. Ding, S. J. Xia, V. I. Birss, J. Electroanal. Chem. 459 (1998) 45

[16] G. Zotti, S. Cattarin, N. Comisso, J. Electroanal. Chem. 239 (1988) 387

[17] V. F. Ivanov, O. L. Gribkova, S. V. Novikov, A. A. Nekrasov, A. A. Isakova, A. V. Vannikov, G. B. Meshakov, I. V. Yamisky, Synth. Met. 152 (2005) 153

[18] M. A. Mohamoud, A. R. Hillman, J. Sol. St. Electrochem. xx (2006) in press

[19] A. Mahmoud, B. Keita, L. Nadjo, J. Electroanal. Chem. 446 (1998) 211

[20] A. R. Hillman, in: A. J. Bard, M. Stratmann (Eds), Encyclopedia of Electrochemistry, 3, Wiley, New York, 2003, p. 230

[21] S. J. Martin, H. L. Bandey, R. W. Cernosek, A. R. Hillman, M. J. Brown, Anal. Chem. 72 (2000) 141

[22] H. L. Bandey, S. J. Martin, R. W. Cernosek, A. R. Hillman, Anal. Chem. 71 (1999) 2205

[23] M. J. Brown, A. R. Hillman, S. J. Martin, R. W. Cernosek, H. L. Bandey, J. Mater. Chem. 10 (2000) 115

[24] A. R. Hillman, A. Jackson, S. J. Martin, Anal. Chem. 73 (2001) 540

[25] R. Lucklum, C. Behling, R. W. Cernosek, S. J. Martin, J. Phys. D: Appl. Phys. 30 (1997) 346

[26] S. J. Martin, V. E. Granstaff, G. C. Frye, Anal. Chem. 63 (1991) 2272

[27] A. R. Hillman, I. Efimov, M. Skompska, Farad. Discuss. 121 (2002) 423

[28] P. A. Kilmartin, G. A. Wright, Electrochim. Acta. 43 (1998) 3091

[29] M. Lapkowski, E. M. Genies, J. Electroanal. Chem. 279 (1990) 157

[30] L. Duic, Z. Mandic, J. Electroanal. Chem. 335 (1992) 207

[31] A. R. Hillman, M. J. Brown, J. Am. Chem. Soc. 120 (1998) 12968

[32] A. Saraswathi, A. R. Hillman, S. J. Martin, J. Electroanal. Chem. 460 (1998) 267

[33] C. Lagier, I. Efimov, A. R. Hillman, Anal. Chem. 77 (2005) 335

[34] H. L. Bandey, A. R. Hillman, M. J. Brown, Farad. Discuss. Chem. Soc. 107 (1997) 105

[35] V. E. Granstaff, S. J. Martin, J. Appl. Phys. 75 (1994) 1319

[36] L. P. Bauermann, P. N. Bartlett, Electrochim. Acta. 50 (2005) 1537 
[37] Y. Harima, R. Patil, K. Yamashita, N. Yamamoto, S. Ito, A. Kitani, Chem. Phys. Lett. 345 (2001) 239

[38] H. Tang, A. Kitani, M. Shiotani, Electrochim. Acta. 41 (1996) 1561

[39] H. Okamoto, T. Kotaka, Polymer 40 (1999) 407

[40] R. Cordova, M. A. d. Valle, A. Arratia, H. Gomez, R. Schrebler, J. Electroanal. Chem. 377 (1994) 75

[41] K. Bade, V. Tsakova, J. W. Schultze, Electrochim. Acta. 37 (1992) 2255

[42] G. M. Morale, M. C. Miras, C. Barbero, Synth. Met. 101 (1999) 686

[43] J. Desilvestro, W. Scheifele, J. Mater. Chem. 3 (1993) 263

[44] D. E. Stilwell, S.-M. Park, J. Electrochem. Soc. 135 (1988) 2254

[45] B. Johnson, S.-M. Park, J. Electrochem. Soc. 143 (1996) 1269

[46] Y. Wei, Y. Sun, X. Tang, J. Phys. Chem. 93 (1989) 4878

[47] A. Arnua, Y. Jimenez, R. Fernandez, R. Torresi, M. Otero, E. J. Calvo, J. Electrochem. Soc. 153 (2006) C455

[48] E. J. Calvo, E. Forzani, M. Otero, J. Electroanal. Chem. 538-539 (2002) 231

[49] S. R. Biaggio, C. L. F. Oliveira, M. J. Aguirre, J. H. Zagal, J. Braz. Chem. Soc. 5 (1994) 203 
Fig.1
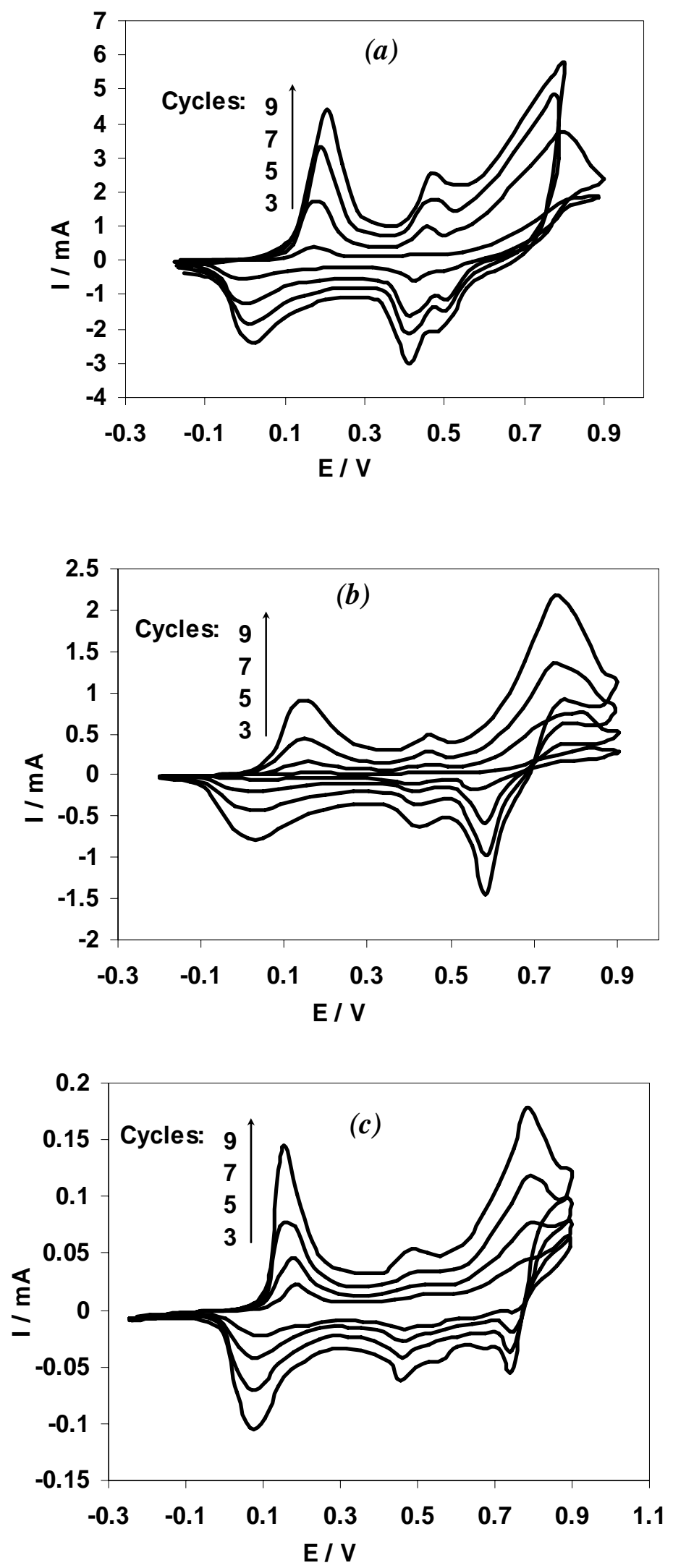

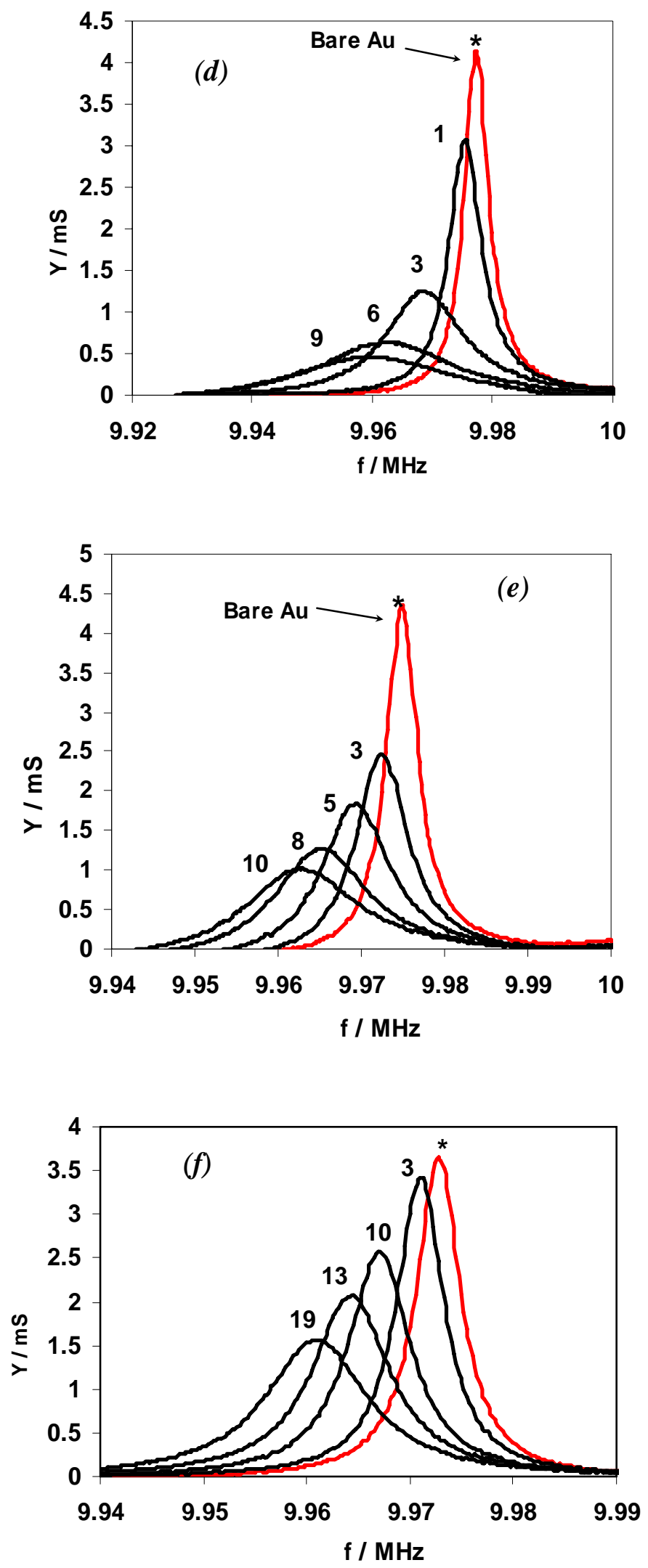
Fig.2
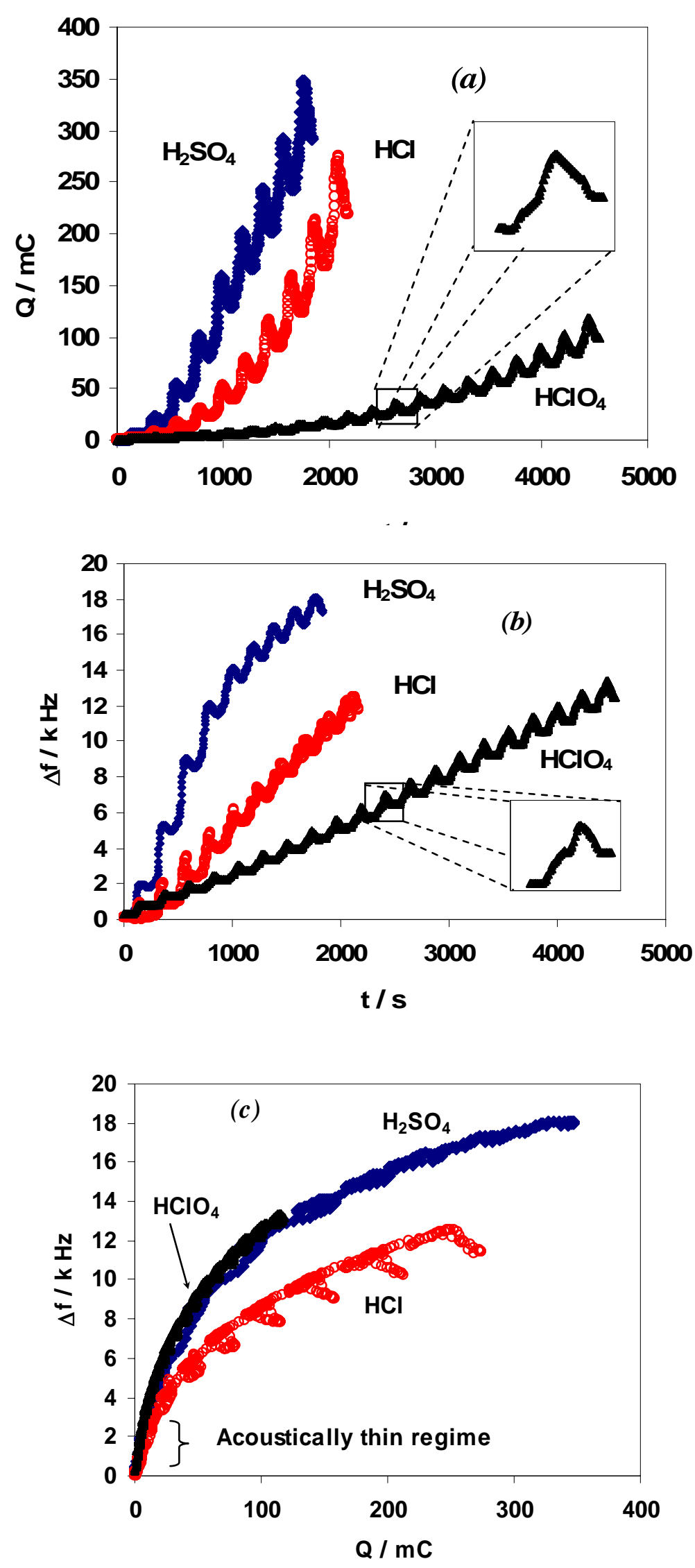
Fig. 3
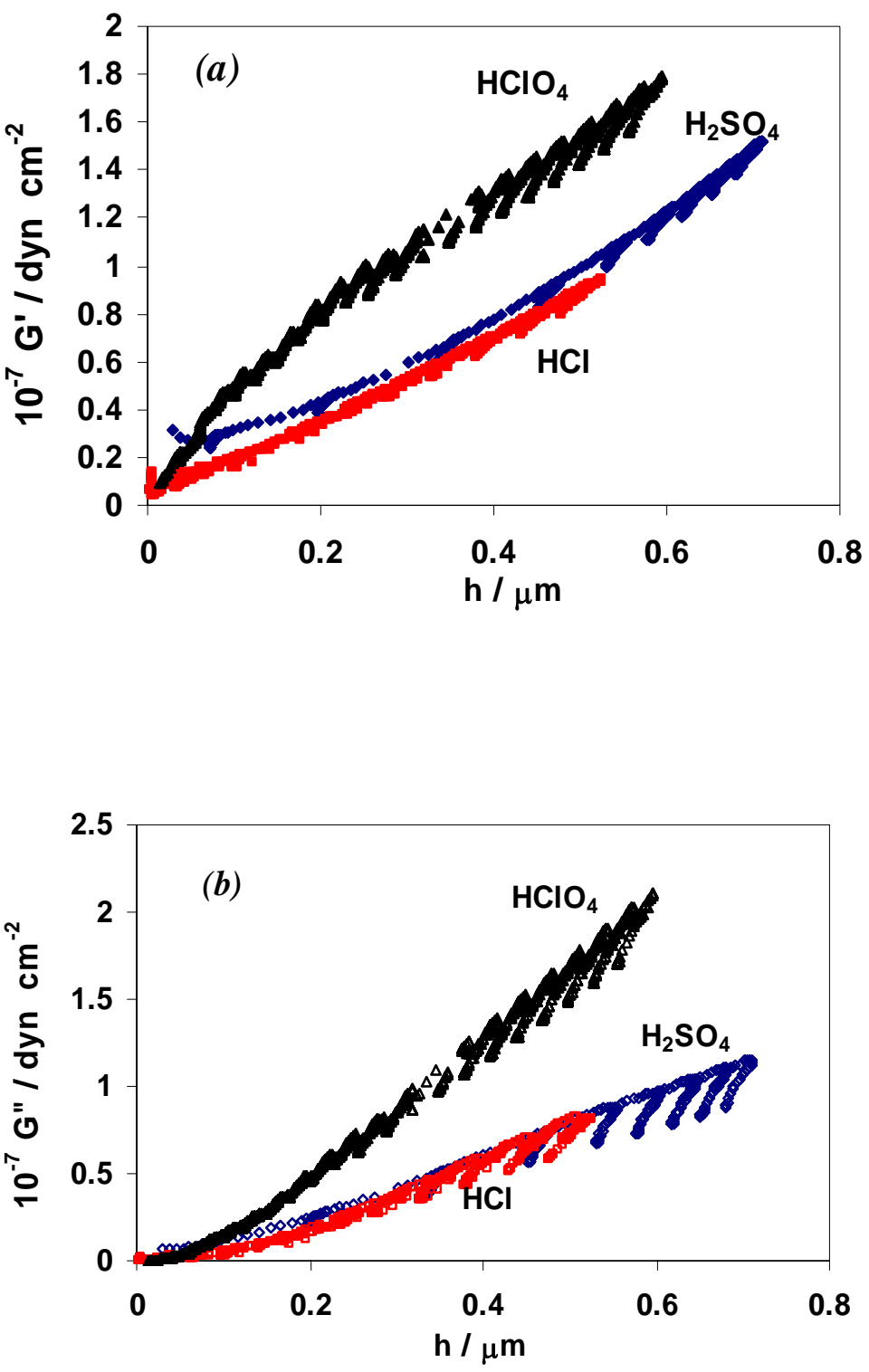
Fig. 4
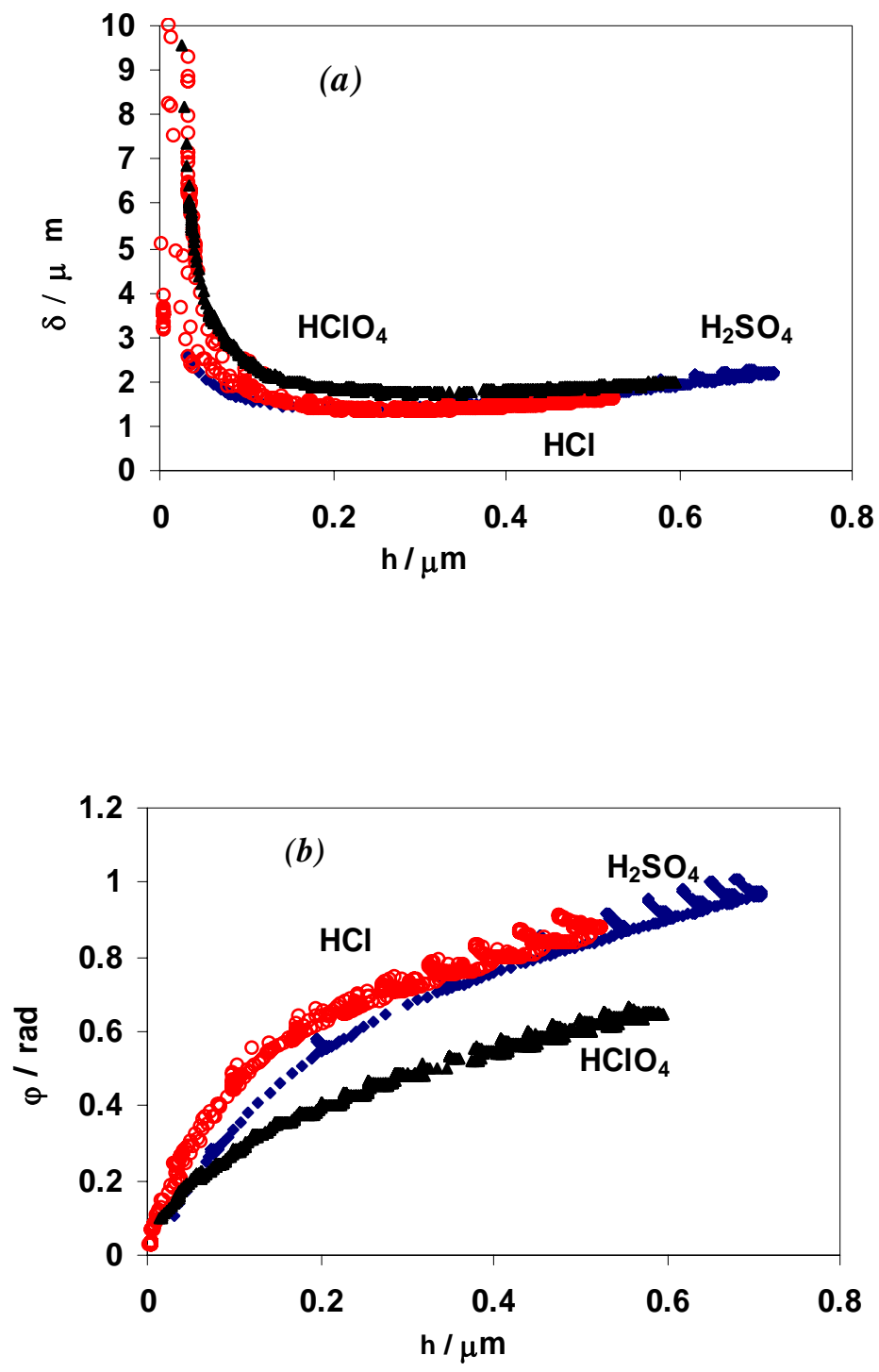
Fig. 5

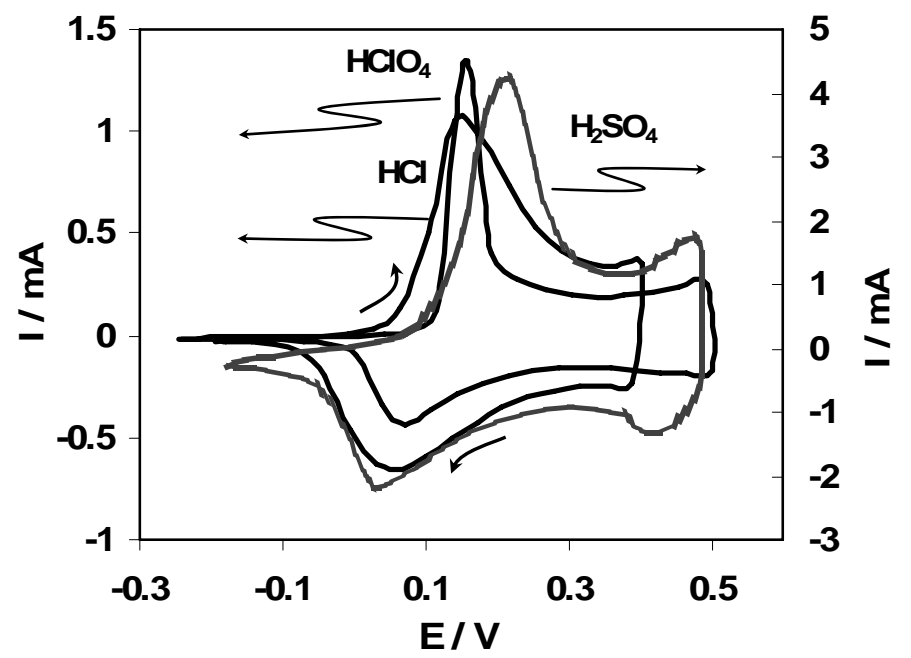

Fig. 6
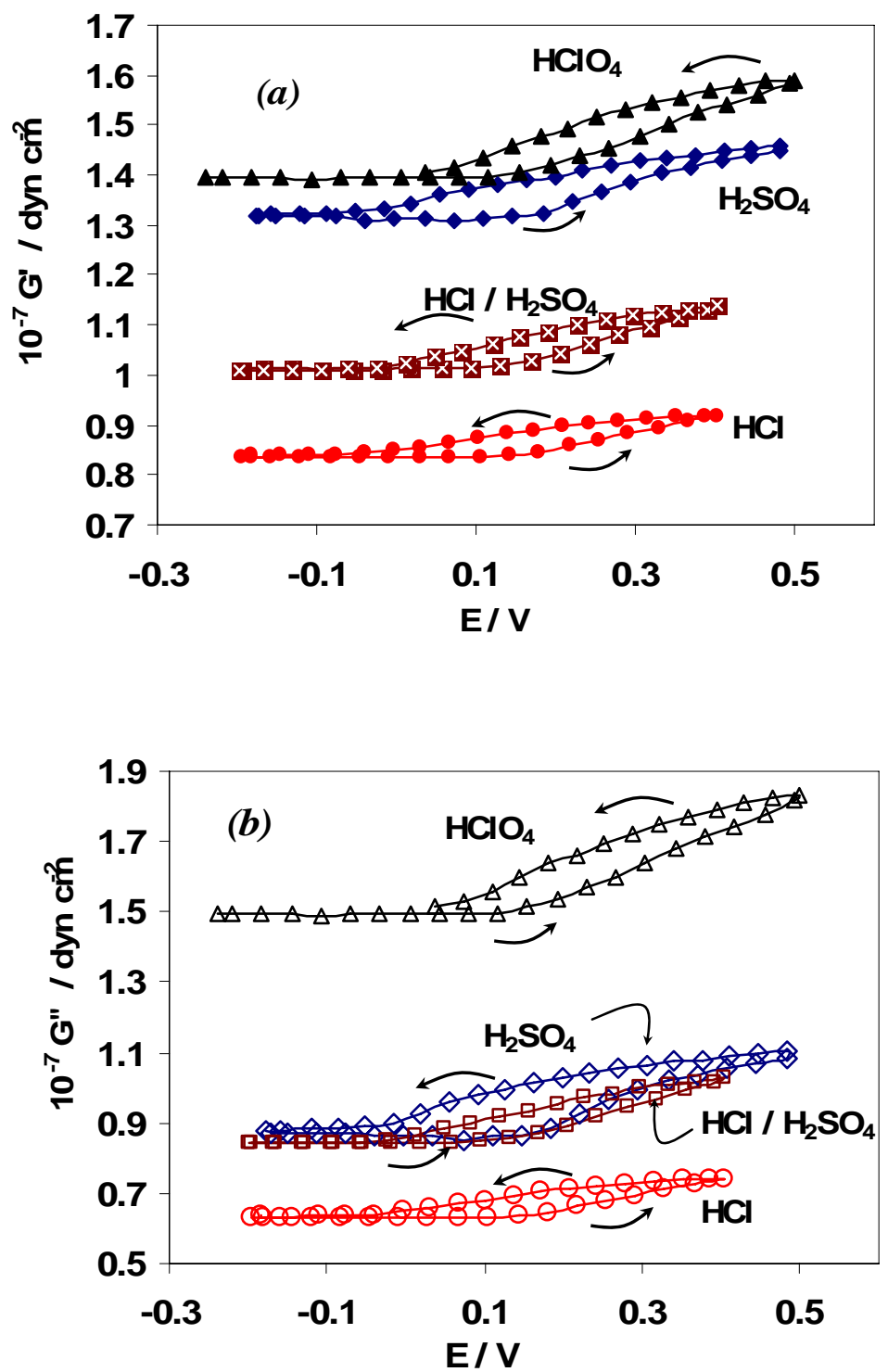
Fig. 7
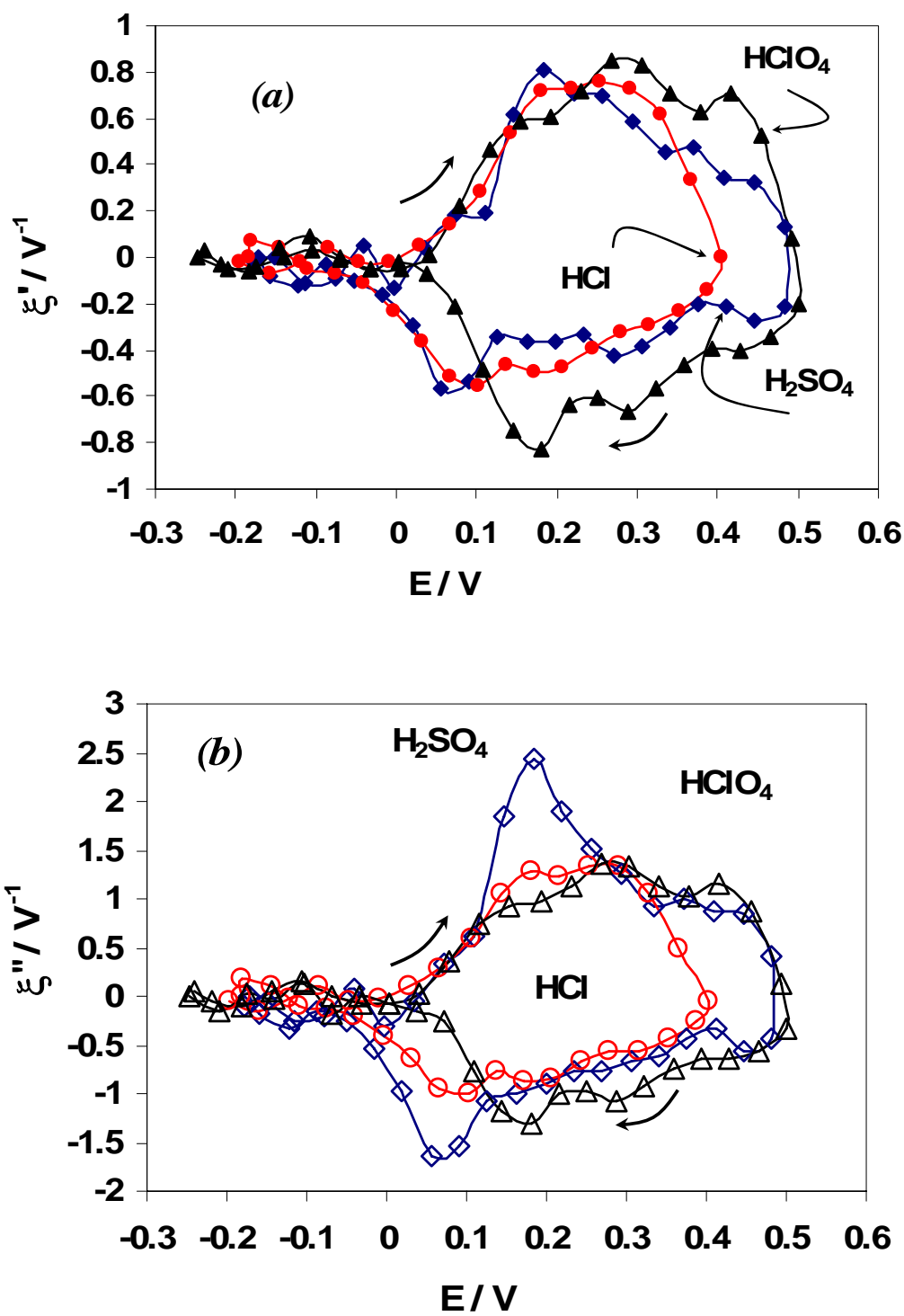